\title{
THE EXISTENCE OF PERIODIC SOLUTIONS TO NONAUTONOMOUS DIFFERENTIAL INCLUSIONS
}

\author{
JACK W. MACKI, PAOLO NISTRI, AND PIETRO ZECCA
}

(Communicated by Kenneth A. Meyer)

\begin{abstract}
For an $m$-dimensional differential inclusion of the form

$$
\dot{x} \in A(t) x(t)+F[t, x(t)],
$$

with $A$ and $F T$-periodic in $t$, we prove the existence of a nonconstant periodic solution. Our hypotheses require $m$ to be odd, and require $F$ to have different growth behavior for $|x|$ small and $|x|$ large (often the case in applications). The idea is to guarantee that the topological degree associated with the system has different values on two distinct neighborhoods of the origin.
\end{abstract}

We prove the existence of a nonconstant $T$-periodic solution to a time-dependent differential inclusion of the form

$$
\dot{x} \in A(t) x(t)+F[t, x(t)] \text { a.e. in }[0, T], T>0 \text { given. }
$$

We assume the following:

(H1) $A: t \mapsto A(t)$ is in $L^{1}\left([0, T], \mathbf{R}^{m \times m}\right) ; F:[0, T] \times \mathbf{R}^{m} \rightarrow 2^{\mathbf{R}^{m}}$ is a $t$-measurable, $x$ upper-semicontinuous multivalued map with nonempty, compact, convex values such that $0 \in F(t, 0)$ a.e. in $[0, T]$, and satisfying

$$
|F(t, x)| \stackrel{\text { dfn }}{=} \max \{|y|: y \in F(t, x)\} \leq \alpha(t)|x|+\beta(t) \quad \text { a.e. in }[0, T]
$$

for some pair $(\alpha, \beta)$ from $L^{1}\left([0, T], \mathbf{R}^{+}\right)$.

(H2) If $0 \in A(t) c+F(t, c)$ a.e. in $[0, T]$ for some fixed $c \in \mathbf{R}^{n}$, then $c=0$.

(H3) We define $i(t, x)=\inf \{(x, A(t) x+y): y \in F(t, x)\}, s(t, x)=$ $\sup \{(x, A(t) x+y): y \in F(t, x)\}$, where $(a, b)$ denotes the inner product of $a$ and $b$ in $\mathbf{R}^{m}$. Assume

$$
\int_{0}^{T} \liminf _{|x| \rightarrow \infty} \frac{i(t, x)}{|x|^{2}} d t>0,
$$

and suppose that there exists an $r>0$ such that $s(t, x)<0$ for all $0<|x|<r$ a.e. in $[0, T]$.

In the following, the maps $A(\cdot)$ and $F(\cdot, x)$ will be considered extended from the interval $[0, T]$ to the real line $\mathbf{R}$ by $T$-periodicity. $\left(L_{T}^{1}\right)^{m}$ is the Banach space of functions in $L^{1}\left([0, T], \mathbf{R}^{m}\right)$, extended to $\mathbf{R}$ by $T$-periodicity. In the same way $\left(A C_{T}\right)^{m}$ and $\left(C_{T}\right)^{m}$ are the Banach spaces of $T$-periodic functions which are absolutely continuous and continuous endowed with the usual norms $\|x\|_{A C}$ and $\|x\|_{\infty}$ respectively.

Received by the editors July 26, 1987 and, in revised form, March 26, 1988. Presented to the Workshop on Ordinary Differential Equations and Differential Inclusions, International School of Advanced Studies, Trieste, May, 1988.

1980 Mathematics Subject Classification (1985 Revision). Primary 34A60, 34C25.

Key words and phrases. Periodic solution, differential inclusions. 
THEOREM. Let the dimension $m$ of the space $\mathbf{R}^{m}$ be odd and let hypotheses (H1)-(H3) hold. Then there exists a nonconstant T-periodic solution of (1).

ProOF. Let $x=x(t)$ be a nontrivial $T$-periodic solution of (1). By hypothesis $x \in(A C)^{m}$. Let us consider

$$
\frac{d}{d t} \frac{|x(t)|^{2}}{2}=(x(t), A(t) x(t)+y(t))
$$

where $y(t) \in F[t, x(t)]$ a.e. in $[0, T]$ is such that $\dot{x}(t)=A(t) x(t)+y(t)$ a.e. in $[0, T]$. We observe that $y \in\left(L_{T}^{1}\right)^{m}$ by (H1). Dividing by $1+|x(t)|^{2}$ and integrating over $[\tau, t], t \in[\tau, \tau+T]$, we get

$$
\frac{1}{2}\left[\log \left(1+|x(t)|^{2}\right)-\log \left(1+|x(\tau)|^{2}\right)\right]=\int_{\tau}^{t} \frac{(x(s), A(s) x(s)+y(s))}{1+|x(s)|^{2}} d s .
$$

If $\tau=0$ and $t=T$ we get

$$
\int_{0}^{T} \frac{(x(s), A(s) x(s)+y(s))}{1+|x(s)|^{2}} d s=0 .
$$

Let us suppose that there exists a sequence of nontrivial $T$-periodic solutions $\left\{x_{n}\right\}$ such that $\left\|x_{n}\right\|_{\infty} \rightarrow 0$ for $n \rightarrow \infty$, that is, $x_{n}(t) \rightarrow 0$ for $n \rightarrow \infty$ uniformly on $[0, T]$. Then for each $n$

$$
\int_{0}^{T} \frac{\left(x_{n}(s), A(s) x_{n}(s)+y_{n}(s)\right)}{1+\left|x_{n}(s)\right|^{2}} d s=0,
$$

and using (H3) for $n$ sufficiently large we get

$$
0=\int_{0}^{T} \frac{\left(x_{n}(s), A(s) x_{n}(s)+y_{n}(s)\right)}{1+\left|x_{n}(s)\right|^{2}} d s \leq \int_{0}^{T} \frac{\left(x_{n}(s), A(s) x_{n}(s)+y_{n}(s)\right)}{1+r^{2}}<0 .
$$

This is a contradiction. Therefore if $x$ is a $T$-periodic solution of (1) and

$$
\max _{t \in[0, T]}|x(t)|<r
$$

then $x(t) \equiv 0$.

Let us prove that there exists $R>0, R>r$, such that if $x$ is a $T$-periodic solution of (1) then $\|x\|_{\infty}<R$.

Suppose the opposite. Then there exists a sequence $\left\{x_{n}\right\}$ of $T$-periodic solutions of (1) such that $\max _{t \in[0, T]}\left|x_{n}(t)\right| \rightarrow+\infty$ for $n \rightarrow \infty$. Using (H1) we get from (**) that for all $n$

$\frac{1}{2}\left[\log \left(1+\left|x_{n}(t)\right|^{2}\right)-\log \left(1+\left|x_{n}(\tau)\right|^{2}\right)\right] \leq \int_{0}^{T}(|A(s)|+\alpha(s)) d s+\int_{0}^{T} \beta(s) d s=M$, where $\tau \in \mathbf{R}$ and $t \in[\tau, \tau+T]$.

By the $T$-periodicity of $x_{n}$ we get

$$
\log \max _{t \in[0, T]}\left(1+\left|x_{n}(t)\right|^{2}\right) \leq \log \min _{t \in[0, T]}\left(1+\left|x_{n}(t)\right|^{2}\right)+2 M,
$$

so $\min _{t \in[0, T]}\left|x_{n}(t)\right|^{2} \rightarrow \infty$ for $n \rightarrow \infty$. This implies $\left|x_{n}(t)\right| \neq 0$ for large $n$, and we can write

$$
\int_{0}^{T} \frac{\left(x_{n}(s), A(s) x_{n}(s)+y_{n}(s)\right)}{\left|x_{n}(s)\right|^{2}} d s=\log \frac{\left|x_{n}(T)\right|}{\left|x_{n}(0)\right|}=0 \quad \text { for } n \text { large. }
$$


Then using (H3) we obtain

$$
\begin{aligned}
0 & =\liminf _{n \rightarrow \infty} \int_{0}^{T} \frac{\left(x_{n}(s), A(s) x_{n}(s)+y_{n}(s)\right)}{\left|x_{n}(s)\right|^{2}} \\
& \geq \int_{0}^{T} \liminf _{|x| \rightarrow \infty} \inf _{y \in F(t, x)} \frac{(x, A(s) x+y)}{|x|^{2}}>0 .
\end{aligned}
$$

This contradicts (H3), so there exists $R>0$ such that $\max _{t \in[0, T]}|x(t)|<R$ for all $T$-periodic solutions of (1).

Let $d / d t, \mathscr{A}:\left(A C_{T}\right)^{m} \rightarrow\left(L_{T}^{1}\right)^{m}$ and $\mathscr{F}:\left(A C_{T}\right)^{m} \rightarrow 2^{\left(L_{T}^{1}\right)^{m}}$ be operators defined by $((d / d t) x)(t)=\dot{x}(t),(\mathscr{A} x)(t)=A(t) x(t)$ and $\mathscr{F}[x](t)=\left\{f \in\left(L_{T}^{1}\right)^{m}: f(t) \in\right.$ $F(t, x(t))$ a.e. in $[0, T]\}$. It is easy to check that the above arguments show that the two homotopies

$$
\begin{gathered}
\frac{d}{d t} x \in \lambda[\mathscr{A} x+\mathscr{F}[x]]+(1-\lambda) I x, \\
\frac{d}{d t} x \in \lambda[\mathscr{A} x+\mathscr{F}[x]]+(\lambda-1) I x, \quad \lambda \in[0,1],
\end{gathered}
$$

where $I$ is the identity on $\left(A C_{T}\right)^{m}$, are admissible homotopies in $B(0, R)$ and $B(0, r)$, respectively, since $\left(O_{1}\right)_{\lambda}-\left(O_{2}\right)_{\lambda}$ do not have any solution on $\partial B(0, R)$ and $\partial B(0, r)$, respectively $\left(B(0, \rho)\right.$ denotes the ball in $\left(C_{T}\right)^{m}$ centered at the origin with radius $\rho>0)$. By $\left(\mathrm{H}_{1}\right)$ there exist $B(0, \bar{R})$ and $B(0, \bar{r})$ in $\left(A C_{T}\right)^{m}$ with the same property. Furthermore, $\left(\mathrm{O}_{1}\right)_{\lambda}-\left(\mathrm{O}_{2}\right)_{\lambda}$ can be rewritten in the equivalent form

$\left(O_{1}\right)_{\lambda}^{\prime}$

$$
\begin{gathered}
x \in \lambda \mathscr{L}^{-1}[\mathscr{A} x+\mathscr{F}[x]-x], \\
x \in \lambda \mathscr{L}^{-1}[\mathscr{A} x+\mathscr{F}[x]+x]-2 \mathscr{L}^{-1} x, \quad \lambda \in[0,1] .
\end{gathered}
$$$$
\left(\mathrm{O}_{2}\right)_{\lambda}^{\prime}
$$

Here $\mathscr{L}^{-1}:\left(L_{T}^{1}\right)^{m} \rightarrow\left(A C_{T}\right)^{m}$ is the inverse of the isomorphism $\mathscr{L}:\left(A C_{T}\right)^{m} \rightarrow$ $\left(L_{T}^{1}\right)^{m}$ defined by $\mathscr{L}[x](t)=\dot{x}(t)-x(t)$ for almost all $t \in[0, T]$. As the immersion $i:\left(A C_{T}\right)^{m} \rightarrow\left(L_{T}^{1}\right)^{m}$ is a compact map, using (H1) we see that the operators

$$
\begin{gathered}
K=\mathscr{L}^{-1}[\mathscr{A}+\mathscr{F}-I] \circ i: B_{T}(0, \bar{R}) \rightarrow 2^{\left(A C_{T}\right)^{m}}, \\
\tilde{K}=\mathscr{L}^{-1}[\mathscr{A}+\mathscr{F}+I] \circ i: B_{T}(0, \bar{r}) \rightarrow 2^{\left(A C_{T}\right)^{m}}
\end{gathered}
$$

are compact upper semicontinuous with convex, compact values (see [4]). Then the topological degree for $\left(O_{1}\right)_{\lambda}^{\prime}$ and $\left(O_{2}\right)_{\lambda}^{\prime}$ is defined in $B(0, \bar{R})$ and $B(0, \bar{r})$ (see [1]). Using in particular the homotopy invariance and normalization properties of degree we see that

$$
\begin{gathered}
d(I-K, B(0, \bar{R}), 0)=d(I, B(0, \bar{R}), 0)=1, \\
d\left(I-\tilde{K}+2 \mathscr{L}^{-1}, B(0, \bar{r}), 0\right)=d\left(I+2 \mathscr{L}^{-1}, B(0, \bar{r}), 0\right)=(-1)^{m} .
\end{gathered}
$$

The second value can be evaluated using the so-called Leray-Schauder formula (see Lloyd [5] for a useful survey on the applications of the topological degree).

Finally, since $(I-K) x=\left(I-\tilde{K}+2 \mathscr{L}^{-1}\right) x$ for all $x \in\left(A C_{T}\right)^{m}$ we have

$$
d(I-K, B(0, \bar{R}), 0)=1 \quad \text { and } \quad d(I-K, B(0, \bar{r}), 0)=(-1)^{m},
$$

so if $m$ is odd, using the additivity property,

$$
d(I-K, B(0, \bar{R}) \backslash B(0, \bar{r}), 0) \neq 0 .
$$


It follows that there exists $x \in B(0, \bar{R}) \backslash B(0, \bar{r})$ such that $0 \in(I-K) x$, that is, a (by (H2) nonconstant) $T$-periodic solution of (1).

REMARK 1. If for each $t \in[0, T], F(t, x)$ is an ordinary single-valued function of $x$ except for a finite number of values $x_{i}(t), i=1, \ldots, k(t), k(t) \leq k$, then it is easy to see that these points have no effect on $i(t, x), s(t, x)$. That is, the multifunction is irrelevant. Thus the multifunction allows us to handle discontinuities with minimal fuss.

REMARK 2. Note that our theorem remains true, with essentially the same proof, if (H3) is replaced by

$$
\int_{0}^{T} \limsup _{|x| \rightarrow \infty} \frac{s(t, x)}{|x|^{2}} d t<0
$$

and there exists $r>0$ such that $i(t, x)>0$ for all $0<|x|<r$ a.e. in $[0, T]$.

REMARK 3. Topological methods similar to those used in this paper were used by the second author to treat control problems under various boundary conditions $[2,6,7]$. The idea of using homotopy to move from one completely continuous functional equation with obvious solution to another goes back (at least) to Krasnosel'skii's work in the 1950's and 1960's (see [3]), that is, the idea is to guarantee that the degree has different values on two neighborhoods of the system.

EXAMPLE 1. Consider the system

$$
\dot{x}=A(t) x+F(t, x), \quad x \in \mathbf{R}^{2 n-1}, n \in N,
$$

satisfying (H1) and (H2), with

$$
F(t, x)= \begin{cases}a_{1}(t) B(x, 1) & \text { for }|x|>\eta, \\ a_{2}(t) B\left(0,|x|^{2}\right) & \text { for }|x|<\rho,\end{cases}
$$

where $\eta>\rho>0$, and $a_{1}, a_{2}$ are positive-valued and in $L^{\infty}([0, T], \mathbf{R})$. Suppose the eigenvalues $\lambda_{i}(t)$ of $A(t)$ are distinct and satisfy

$$
\lambda_{1}(t)<\lambda_{2}(t)<\cdots<\lambda_{2 n-1}(t)<-\alpha^{2}<0 \text { for a.a. } t \in[0, T],
$$

with $\int_{0}^{T}\left|\lambda_{1}(t)\right| d t<\int_{0}^{T} a_{1}(t) d t$. We compute for $|x|>\eta, i(t, x)=x^{t r} A(t) x+$ $a_{1}(t)\left[|x|^{2}-|x|\right]$, while for $0<|x|<\rho, s(t, x)=x^{t r} A(t) x+\left|a_{2}(t)\right||x|^{3}$. It is easy to see that our theorem applies.

EXAMPLE 2. Consider the system $\dot{x} \in A(t) x+F(x), x \in \mathbf{R}^{2 n-1}, n \in N$,

$$
F(t)= \begin{cases}-x /|x|, & x \neq 0 \\ B(0,1), & x=0 .\end{cases}
$$

In one dimension this is a standard "two constant values with a jump at the origin" system. Suppose the eigenvalues $\lambda_{i}(t)$ of $A(t)$ are distinct and satisfy $\lambda_{1}(t)<$ $\lambda_{2}(t)<\cdots<\lambda_{2 n-1}(t)$ for a.a. $t \in[0, T]$. If $\int_{0}^{T} \lambda_{1}(t) d t>0$ then our theorem applies. In fact (H1) and (H2) clearly hold and by computation we obtain

$$
s(t, x)=i(t, x)=x^{t r} A(t) x-|x|, \quad x \neq 0,
$$

so it is easy to see that (H3) holds.

EXAMPLE 3. The theorem fails if $m$ is even. The following elegant and simple counterexample was provided by the referee. Consider the 2-dimensional system

$$
\begin{aligned}
& x^{\prime}=-\alpha x \cos w(x, y)+\alpha y \sin w(x, y), \\
& y^{\prime}=-\alpha x \sin w(x, y)-\alpha y \cos w(x, y),
\end{aligned}
$$


with $w(x, y)=2 \arctan \left(x^{2}+y^{2}\right), \alpha>0$ a real constant such that $2 \pi / \alpha \neq T / n$ for any natural number $n$. Notice that if $\rho^{2}(t) \equiv x^{2}(t)+y^{2}(t)<1$ then $(d / d t)\left(\rho^{2}\right)<0$, while if $\rho^{2}(t)>1$, then $(d / d t)\left(\rho^{2}\right)>0$. If $\rho^{2}(t)=1$, then $x^{\prime}(t)=\alpha y(t), y^{\prime}(t)=-\alpha x(t)$, so $(x, y)$ will give a periodic solution with period $2 \pi / \alpha$. Clearly there do not exist nontrivial solutions of period $T$.

ACKNOWLEDGMENT. We wish to thank the referee for his/her useful suggestions.

\section{REFERENCES}

1. A. Cellina and A. Lasota, $A$ new approach to the definition of topological degree for multivalued mappings, Atti Accad. Naz. Lincei Rend. Cl. Sci. Fis. Mat. Natur. (8) 47 (1969), 434-440.

2. M. Furi, P. Nistri, M. P. Pera, and P. L. Zezza, Topological methods for the global controllability of nonlinear systems, J. Optim. Theory Appl. 45 (1985), 231-255.

3. M. A. Krasnosel'skii and P. P. Zabreǐko, Geometrical methods of nonlinear analysis, SpringerVerlag, New York, 1984.

4. J. M. Lasry and R. Robert, Analyse nonlineaire multivoque, Cahiers de Math. de la Decision \#7611, Equipe de Recherche de Math. de la Decision, Equipe de Recherche Associe de CNRS \#249, Ceremade.

5. N. G. Lloyd, A survey of degree theory: Basis and development, IEEE Trans. Circuits and Systems CAS 30 (1983), 607-616.

6. P. Nistri, Periodic control problems for a class of nonlinear periodic differential equations, Nonlinear Anal. 7 (1983), 79-90.

7. __ Nonlinear boundary value control problems, Proc. 25th IEEE Conf. on Decision and Control, Athens, Dec. 1986, pp. 600-601.

Department of Mathematics, University of Alberta, Edmonton, Alberta, CANADA T6G 2G1 (Current address of Jack W. Macki)

Dipartimento di Sistemi e INFORMATICA, FACOlTÁ di INGEgNERIA, UNIVERSiTÀ Di FIRENZE, VIA Di S. MARTA 3, 50139 FIRENZE (FlORENCE), ITALY (Current address of Paolo Nistri and Pietro Zecca) 